\title{
INFECTION CONTROL IN ALTERNATIVE HEALTHCARE SETTINGS
}

\author{
Elaine Flanagan, RN BSN MSA CIC ${ }^{1}$, Teena Chopra, MD², and Lona Mody, MD, M.Sc. ${ }^{3}$ \\ ${ }^{1}$ Department of Infection Prevention and Hospital Epidemiology, Detroit Medical Center, Veterans \\ Affairs Ann Arbor Healthcare System \\ ${ }^{2}$ Division of Infectious Diseases and Infection Control, Wayne State University, Veterans Affairs \\ Ann Arbor Healthcare System \\ ${ }^{3}$ University of Michigan Medical School, Division of Geriatric Medicine and Geriatrics Research, \\ Education and Clinical Center, Veterans Affairs Ann Arbor Healthcare System
}

\section{SYNOPSIS}

With the changing healthcare delivery, patients receive care at various settings including acute care hospitals, skilled nursing facilities, outpatient primary care and specialty clinics, as well as at home, exposing them to pathogens in various settings.

Various healthcare settings face unique challenges requiring individualized infection control programs.

Infection control programs in skilled nursing facilities should address: surveillance for infections and antimicrobial resistance, outbreak investigation and control plan for epidemics, isolation precautions, hand hygiene, staff education, and employee and resident health programs.

Infection control programs in ambulatory clinics should address: Triage and standard transmission based precautions, cleaning, disinfection and sterilization principles, surveillance in surgical clinics, safe injection practices, and bioterrorism and disaster planning for ambulatory clinics.

\section{BACKGROUND}

Healthcare delivery in the United States has evolved significantly over the latter part of twentieth century. Healthcare delivery has moved from acute care facilities to skilled nursing facilities (SNF), rehabilitation units, assisted living, home, and outpatient settings. Measures to reduce healthcare costs have led to a reduced number of hospitalizations and shorter lengths of stay (with an increase in the severity of illness among hospitalized patients including more frequent ICU admissions), along with increased outpatient, home care, and SNF stays for older adults. ${ }^{1-3}$

This review focuses on infection control issues in skilled nursing facilities and ambulatory clinics.

Contact Author, Lona Mody, MD, 11-G GRECC, AAVAMC, 2215 Fuller Dr, Ann Arbor, MI 48105, Phone: 7348453072 , lonamody@umich.edu.

Publisher's Disclaimer: This is a PDF file of an unedited manuscript that has been accepted for publication. As a service to our customers we are providing this early version of the manuscript. The manuscript will undergo copyediting, typesetting, and review of the resulting proof before it is published in its final citable form. Please note that during the production process errors may be discovered which could affect the content, and all legal disclaimers that apply to the journal pertain. 


\section{INFECTION PREVENTION PROGRAMS IN SKILLED NURSING FACILITIES}

Skilled nursing facilities are emerging as a major healthcare delivery site.

Approximately 1.43 million older adults reside in Centers for Medicare and Medicaid (CMS) certified SNFs (2006 data). About 3\%-15\% of such patients acquire an infection in these facilities. In a year, there are approximately 2.1 million discharges from SNFs with the primary reasons for discharge being death or transfers to hospitals. These numbers are expected to grow as the population ages. ${ }^{2}$

Skilled nursing facilities provide of two distinct types of care for older patients: a) long term care for older adults with irreversible functional and cognitive deficits and b) sub-acute care for patients who require a short admission to complete their medical treatment plan and to regain their functional strength before returning to their independent living.

As SNFs accept increasingly complex patients from acute care, infection prevention becomes crucial. Infection prevention research in the SNF setting has made enormous strides in the last two decades. There is an increasing recognition that infection prevention strategies have to be more individualized than in hospital settings and residents' social well being remains paramount.

However, skilled nursing facilities have unique characteristics that create special challenges in implementing an effective infection prevention program. First, skilled nursing facility residents are particularly susceptible to infections because of multi-morbidity, greater severity of illness, functional impairment, cognitive impairment, incontinence, and the presence of frequent short term and long-term indwelling device use such as urinary catheters and feeding tubes. Second, skilled nursing facility residents may also serve as host reservoirs for antimicrobial-resistant pathogens such as MRSA and VRE. With reduction in the length of hospital stay, the severity of illness among residents of the subacute care nursing unit has increased with resultant inherent rapid transfers to a hospital. Older adults serve as vectors, transmitting pathogens from one setting to another. Third, utility of diagnostic specimens is often limited due to difficulty in obtaining specimens from older adults (such as a clean catch urine sample or a sputum sample) and lag time between specimen acquisition and clinical evaluation. Conversely, some tests might be more frequently obtained and might lead to inappropriate antibiotic usage. All of these factors combined create an environment where vulnerable residents are highly prone to infections and acquisition of resistant pathogens. The diagnostic dilemmas often lead to suboptimal management of infections in older adults and make infection prevention programs even more crucial.

\section{INFECTION PREVENTION PROGRAM IN SKILLED NURSING FACILITIES: FUNCTIONS, COMPONENTS AND OVERSIGHT}

Main functions for an infection prevention program include: a) obtaining and managing critical data including surveillance information for endemic infections and outbreaks; b) developing and updating policies and procedures; c) developing individualized interventions to prevent infections and antimicrobial resistance; and d) educating and training HCWs, patients, visitors, and non medical caregivers. ${ }^{1}$

An effective infection prevention program includes a method of surveillance for infections and antimicrobial-resistant pathogens, an outbreak control plan for epidemics, isolation and standard precautions, hand hygiene, staff education, an employee health program, a resident health program, policy formation and periodic review with audits, and a policy to 
communicate reportable diseases to public health authorities. An infection preventionist is crucial in developing and executing an infection prevention program.

\section{Information transfer during care transitions}

Transitional care is defined as "a set of actions designed to ensure the coordination and continuity of health care as patients transfer between different locations or different levels of care in the same location". ${ }^{4}$ For older adults, these locations can include acute care hospitals, nursing homes, skilled nursing facilities, rehabilitation units, assisted living facilities, inpatient hospice care, home care, and out-patient primary and specialty clinics. During these care transitions, older adults are particularly prone to fragmented care which can lead to errors and omissions in healthcare delivery. These transitions also provide an opportunity of pathogens to be transferred from one setting to another. It then becomes important for the SNFs to request clinical information from the transferring facility regarding current culture reports of the resident's body sites that may be infected or colonized with pathogenic organisms, especially multi-drug resistant organisms. This action will enable the physician providers and healthcare workers to determine the nursing care interventions necessary to meet the resident's needs and to prevent spread of pathogens in the facility.

\section{Hand Hygiene}

Hand hygiene remains the most effective and least expensive measure to prevent transmission of pathogenic organisms in healthcare setting. Despite calls from numerous local, national and international organizations and infection prevention societies, compliance with hand hygiene remains dismal averaging only $30 \%-50 \% .^{5-8}$ Reasons frequently reported for poor compliance with hand hygiene measures by HCWs include skin irritation from frequent washing, too little time due to a high workload, and simply forgetting. Hand hygiene is discussed in details in Chapter XXXX.

The World Health Organization (WHO) launched its first Global Patient Safety Challenge, 'Clean Care is Safer Care,' in October 2005. ${ }^{9}$ The objective of this initiative is to reduce healthcare-associated infections around the globe. The program's initial focus is the promotion of hand hygiene practices in diverse healthcare systems. WHO has also developed an innovative core theme, 'My five moments of hand hygiene,' which details appropriate situations for compliance with hand hygiene measures during healthcare delivery. This approach provides guidelines and specific recommendations to enhance hand hygiene compliance and is targeted at a broad audience of healthcare workers, hospital administrators, and health authorities.

The use of waterless, alcohol-based handrubs as an adjunct to washing hands with soap and water has become a routine practice by HCWs in many acute care facilities. ${ }^{10}$ Introduction of alcohol-based handrubs has been shown to significantly improve hand hygiene compliance among HCWs in acute care hospitals and to decrease overall nosocomial infection rates and transmission of MRSA infections. Alcohol-based handrubs have also been shown to enhance compliance with hand hygiene in SNFs and should be used to complement educational initiatives. ${ }^{6}$ While the cost of introducing alcohol-based handrubs could be a concern of SNFs, recent data in acute care have shown that the total costs of a hand hygiene promotion campaign including alcohol-based handrub corresponded to less than $1 \%$ of costs that could be attributed to nosocomial infections. ${ }^{7}$

While introducing alcohol-based hand rub in healthcare settings is a prudent, cost-effective measure, several issues need to be considered. Alcohol-based handrubs should not be used if hands are visibly soiled, in which case hand hygiene with antimicrobial soap and water is recommended. Alcohol-based hand rubs can cause dryness of skin; however, recent data on 
rubs containing emollients have shown that hand rubs cause significantly less skin irritation and dryness than soap and water. ${ }^{11}$ Facilities should be aware that alcohols are flammable. Facilities have reported difficulties in implementing the current hand hygiene guidelines pertaining to the use of alcohol-based handrubs due to the fire-safety concerns. Existing national fire codes permit use of alcohol-based handrub dispensers in patient rooms but not in egress or exit corridors. Since the state and local fire codes may differ from national codes, facilities should work with their local fire marshals to insure that installation of alcohol-based handrub containers is consistent with local fire codes.

\section{Multi-drug Resistant Organisms}

Infection and colonization with antimicrobial-resistant pathogens are important concerns in SNFs and develops primarily due to widespread use of empiric antibiotics, functional impairment, use of indwelling devices, mediocre adherence to hand hygiene programs among HCWs, and cross-transmission during group activities. A SNF can reduce infections and colonization with resistant pathogens by emphasizing hand hygiene, developing an antimicrobial utilization program, encouraging evidence-based clinical evaluation and management of infections, and ensuring that the facility has a well-established individualized infection prevention program. Guidelines and expert reviews to control MRSA and VRE provide a good base for developing facility-specific policies. ${ }^{1,12-14}$

There has been some debate on the role of active surveillance cultures (cultures used to detect asymptomatic colonization of a patient with an organism) and their impact on isolation policies in acute care hospitals. The SHEA guideline for preventing nosocomial transmission of multi-drug resistant organisms advocates for use of active surveillance cultures, ${ }^{15}$ whereas the recent draft of HICPAC guidelines call for individual facilities to assess their own needs and conduct surveillance cultures as they deem necessary.16 These guidelines refer to studies from acute care hospitals serving a sicker, shorter-stay population than those served by SNFs. The role of active surveillance cultures in SNFs has not been clearly defined. Facilities should evaluate these guidelines and individualize their plan to obtain active surveillance cultures based on the population they serve, baseline rates of MRSA and VRE, and any recent outbreaks.

\section{Isolation Precautions}

The Centers for Disease Control and Prevention's Healthcare Infection Control and Prevention Advisory Committee (HICPAC) has proposed a two-tiered structure for isolation precautions. In the first tier, HICPAC proposes use of "Standard Precautions," which have been designed for the care of all patients in hospitals, regardless of their diagnosis, infectious or otherwise. Standard precautions constitute the primary strategy for preventing transmission of organisms between patients and healthcare workers. In the second tier are "Transmission-based Precautions," which have been designed for the care of patients suspected of or known to be infected with epidemiologically important pathogens that have been acquired by physical contact or airborne or droplet transmission. ${ }^{17}$

Standard precautions apply to blood, all body fluids, secretions and excretions regardless of whether they contain visible blood, non-intact skin, and/or mucous membrane material. Designed to reduce the risk of transmission of pathogens, both from apparent and ambiguous sources of infection, these precautions include hand hygiene compliance, glove use, masks, eye protection, and gowns, as well as avoiding injuries from sharps. Two new elements of standard precautions added in the new HICPAC isolation precautions guidelines include respiratory hygiene/cough etiquette and safe injection practices also apply to skilled nursing facilities. The elements of respiratory hygiene/cough etiquette includes education of healthcare facility staff, patients and visitors; use of appropriate language and education 
displayed on posters and signs; source control measures such as covering the mouth/nose with a tissue and prompt disposal of used tissues; hand hygiene after contact with respiratory secretions and spatial separation of symptomatic patients of $>3$ feet in common areas if possible. Safe injection practices include the use of single-use, sterile, disposable needle and syringe for each injection and prevention of contamination of any injection equipment and medication. ${ }^{17}$

Transmission-based precautions are intended for use with patients who may be infected with highly transmissible or epidemiologically significant pathogens. These include airborne precautions (e.g., for tuberculosis), droplet precautions (e.g., for influenza), and contact precautions (e.g., for Clostridium difficile). Although these guidelines were designed for acute care settings, several of them, especially the universal precautions, apply to SNFs as well. These recommendations have to be adapted to the needs of the individual facility. A single patient room is preferred for residents who require contact precautions. Often SNFs have high occupancy rates and a paucity of single-resident rooms. In such situations, residents should be cohorted or kept with the same room mate. Spatial separation of $\geq 3$ feet between beds is advised to reduce the opportunities for inadvertent sharing of items between a colonized/infected patient and an uncolonized patient. ${ }^{17}$

Private rooms are generally indicated for residents with uncontrolled excretions (diarrhea), secretions, excessive coughing, heavy wound drainage or widespread skin disease. Singleresident rooms are preferable while the above conditions are active. However, if no private rooms are available, the resident can be placed in a semi-private room, preferably with a resident who is at low risk of developing an infection (such as one who is well nourished, ambulatory, can perform daily activities independently and has no indwelling catheters/lines or open wounds).

Residents, colonized or infected with a specific pathogen, should participate in facility activities and eat in the dining hall. Since there are both recognized and unrecognized pathogen carriers participating in these group activities, all residents should have wounds or invasive sites cleansed, covered, and have hands washed before leaving their rooms.

\section{Surveillance for Infections and Antimicrobial Usage}

Infection surveillance in SNFs involves collection of data on facility-acquired infections. Surveillance is defined as "ongoing, systematic collection, analysis, and interpretation of health data essential to the planning, implementation, and evaluation of public health practice, closely integrated with timely dissemination of these data to those who need to know". ${ }^{18}$ Surveillance can be limited based on a particular objective, a particular ward, an unusual organism or may be facility-wide.

For surveillance to be conducted correctly, utilization of standardized SNF appropriate definitions of infections are crucial.19,20 Besides using valid surveillance definitions, a facility must have clear goals and aims for setting up a surveillance program. These goals, as with other elements of an infection control program, have to be reviewed periodically to reflect changes in the facility's population, pathogens of interest, and changing antimicrobial resistance patterns. Plans to analyze the data and use of these data to design and implement proven preventive measures must also be clearly delineated in advance. The analysis and reporting of infection rates in SNFs are typically conducted monthly, quarterly, and annually to detect trends. Infection rates (preferably reported as infections/1,000 resident-days) can be calculated by using as the denominator resident-days or average resident census for the surveillance period. 
These data can then been used to establish endemic baseline infection rates, and to recognize variations from the baseline that could represent an outbreak. This information should eventually lead to specific, targeted infection control initiatives and to evaluate the success of the changes. Additionally, a facility's surveillance system should include monitoring for appropriate antibiotic use. For example positive culture in a person without clinical symptoms rarely requires treatment with antibiotics.

\section{Outbreak Management}

An illness in a community or region is considered an outbreak when the frequency of this illness clearly exceeds the normal rate of expectancy. The existence of an outbreak is thus always defined relative to the number of cases that are expected to occur in a specific population in a specific time period.

The main objectives of an outbreak investigation are control and elimination of the source, prevention of new cases, prevention of future outbreaks, research to gain additional knowledge about the infectious agent and its mode of transmission, program evaluation and strategies for improvement.

It is vital that the IP, in conjunction with physician support, has the skills to recognize an outbreak, conduct appropriate data collection methods, analyze and interpret the data using simple epidemiologic measures, conduct an initial outbreak investigation efficiently and institute emergent, effective and appropriate outbreak control measures. While local health departments are available for counseling, it may also be beneficial for the IP to have access to a hospital epidemiologist for consultation.

\section{Rehabilitation Services}

Skilled nursing facilities increasingly are responsible for post-acute care rehabilitation, including physical therapy (PT), occupational therapy (OT), and wound care with or without hydrotherapy. These therapists, like other clinical staff such as nurses and nurses' aides, frequently come into contact with residents and thus have many opportunities to transmit pathogens. In a SNF, PT and OT services can be provided either at the bedside or in a central therapy unit. For bedside therapy, therapists may move between rooms and units and do not routinely wear gloves and gowns. For care at a central therapy unit, residents are transported to an open unit, where handwashing sinks may not be readily available. While therapists have not been implicated in any major outbreaks, hydrotherapy for wounds has been shown to facilitate outbreaks with resistant pathogens. ${ }^{21}$

A detailed infection control program for rehabilitation services should be prepared and focus on facility design to promote hand hygiene compliance including convenient and easy access to sinks and the use of alcohol-based handrubs. Patients who are infectious should not be treated at the central facility. Facilities providing hydrotherapy should consider providing the service in a dedicated room with a separate resident entrance.

\section{Resident and Employee Health Program}

The resident health program should focus on immunizations, tuberculin testing, and infection control policies to prevent specific infections. The program should include areas such as skin care, oral hygiene, prevention of aspiration, and catheter care to prevent urinary tract infections. Adults over the age of 65 should receive pneumococcal vaccination at least once, influenza vaccination every year, and a tetanus booster every ten years.

The employee health program mainly concerns employees with potentially communicable diseases, policies for sick leave, immunizations, and OSHA regulations to protect them from 
blood-borne pathogens. It is a requirement that SNFs bar employees with known communicable diseases or infected skin lesions from providing direct contact with the residents, and that employees with infected skin lesions or infectious diarrhea be prevented from having direct contact with residents' food. Moreover, when hiring new employees, an initial medical history must be obtained along with a physical examination and screening for tuberculosis.

Infection control policies and measures in SNFs must be in place to address post-exposure prophylaxis for infections such as HIV and hepatitis B. Varicella vaccine should be given to employees not immune to the virus. Employees are expected to be up-to-date with their tetanus boosters and to receive influenza vaccinations every year. Not only is the influenza vaccine effective in preventing influenza and reducing absenteeism in HCWs, it has also been associated with a decrease in influenza mortality in residents. ${ }^{25}$ Annual influenza vaccination campaigns play a central role in deterring and preventing nosocomial transmission of the influenza virus and should be promoted by the IP and SNF leadership.

\section{Role of Infection Preventionist}

An infection preventionist (IP), usually a staff nurse, is assigned the responsibility of directing infection control activities in a SNF. The IP is responsible for implementing, monitoring, and evaluating the infection control program. Often an IP also functions as an assistant director of nursing, or is involved in staff recruitment and education. For an infection control program to succeed, the IP should be empowered with sufficient time and resources to carry out infection control activities. The IP should also be familiar with the federal, state, and local regulations regarding infection control. Collaborating with an infectious diseases epidemiologist should be encouraged. Such collaborations could also provide assistance with outbreak investigations, emergency preparedness in the event of bioterrorism and vaccine shortages, and the use of microbiologic and molecular methods for infection prevention. Resources available to the IP are discussed in Table 1.

\section{Environmental Rounds}

The IP should conduct walk-rounds on a regular basis to make observations regarding equipment decontamination, and cleaning procedures in bathroom/tub areas; and adherence to infection control guidelines by physical therapy, in medication/treatment rooms, and in kitchen and laundry areas. Observations should be made regarding availability of soap and paper towels, handling of sharps and infectious waste and storage of healthcare supplies, medications and food.

\section{Staff Education}

The IP plays a vital role in meeting these requirements and in educating SNF personnel on various infection control measures, particularly in view of rapid staff turnover that occurs at many SNFs. Informal education during infection control and quality improvement meetings as well as during infection control walk rounds should be complemented with in-service education on hand hygiene, appropriate and early diagnosis of infections, indications for antibiotic usage and antimicrobial resistance, and isolation precautions and policies.

Ongoing staff education is important due to the new research and guidelines published every year, advancements in technology, and regulatory demands. The Joint Commission on Accreditation of Healthcare Organizations (JCAHO) expects new employee orientation to include the facility's infection prevention program; and the employee's individual responsibility is to prevent infections. In addition, the Occupational Safety and Health Administration (OSHA) requires training for blood-borne pathogens and tuberculosis for any employee expected to come into contact with potentially infectious agents. 


\section{Oversight Committee}

The Federal Nursing Home Reform Act from the Omnibus Budget Reconciliation Act of 1987 mandated the formation of a formal infection control committee to evaluate infection rates, implement infection control programs, and review policies and procedures. This mandate has been dropped by OBRA at the federal level. However some states may still require them. A small sub-committee or a working group comprised of the medical director, administrator or nursing supervisor, and ICP should evaluate the SNF infection rates on a regular basis and present the data at quality control meetings, review policies and any relevant research, and make decisions regarding infection control changes. This subcommittee can review and analyze the surveillance data, assure that this data are presented to the nursing and physician staff, and approve targeted recommendations to reduce the incidence of infections. Records pertaining to these activities and infection data should be kept and filed for future reference.

Principles guiding infection control practices also provide a model for enhancing quality of care and patient safety for other non-infectious adverse outcomes such as falls, delirium, inappropriate medication usage, and adverse drug events.

\section{AMBULATORY CARE CENTERS}

Ambulatory care settings have the same infection prevention and control requirements as inpatient hospital settings, but the method of application to comply with the standards will vary depending on the type of care provided by that clinic to their population. The settings range from clinics that provide medical specialty expertise to clinics that provide invasive procedures such as hemodialysis, endoscopy and surgical centers. There are many challenges in ambulatory care to reduce infection risk and improve patient safety. Infection control oversight and accountability is often lacking, especially if the clinics are not part of a hospital or system.

\section{Communicable Disease and Isolation Management}

Patients may be exposed to infectious diseases in the ambulatory waiting rooms. Healthcare workers need to use Standard Precautions in the care of all patients, but patients exhibiting communicable disease symptoms should be managed per the Centers for Disease Control and Prevention (CDC) Isolation Precautions. ${ }^{17}$ Since most ambulatory centers do not have adequate private rooms, a triage policy should be followed. The goal is to promptly identify and process patients with symptoms compatible with communicable disease in order to protect other patients and ambulatory care staff from exposure or infection. Symptoms of a communicable disease may include tuberculosis symptoms such as cough, bloody sputum, night sweats, weight loss, anorexia, and fever. Symptoms of other communicable diseases might include rash of unknown origin or persistent cough. Patients with respiratory symptoms should wear a mask and should be promptly processed to a separate waiting area or examination room apart from other patients. If the ambulatory site has a negative pressure room for AFB and Airborne isolation, patients with suspected tuberculosis and other communicable diseases transmitted by the airborne route should be triaged to these rooms as soon as possible. 22 When entering the room of a patient with suspected or confirmed tuberculosis, all ambulatory staff should wear a fit tested particulate respirator. Cough inducing procedures such as aerosol administration of medication, bronchoscopy and sputum inductions on patients with a respiratory illness poses a high risk of aerosolization of organisms and infection of staff and other patients. Rooms where these aerosol-producing procedures are performed must be modified to meet Airborne isolation standards to protect ambulatory care staff and other patients. Ambulatory surgery centers must provide particulate respirators to staff caring for patients maintained in AFB and Airborne isolation. 
Dental clinics should defer treatment on a patient with known or suspected Mycobacterium tuberculosis or other potentially communicable airborne disease unless it is determined that the procedure is an emergency.

Respiratory Hygiene/Cough Etiquette is an infection control component of the CDC Standard Precautions in care of patients. The protocol is optimized by giving patients tissues and instructions to cover their mouth and nose when coughing or sneezing and disposing of tissues in the trash. Handwashing must be reinforced with accessible handwashing facilities or waterless hand hygiene agents. Patients with a persistent cough are to be given a surgical mask while in a waiting room or other common areas and instructed to ask for a new mask if their old mask becomes soiled or moistened. Respiratory Etiquette signage is a Joint Commission requirement for ambulatory centers within hospital institutions, but is encouraged to be used in all ambulatory centers especially during flu season.

\section{Environmental Hygiene- Cleaning Disinfection and Sterilization}

Cleaning and disinfection of frequently touched surfaces reduces the risk of transmission of multiple drug resistant organism and Clostridium difficile. Ambulatory settings need to standardize cleaning procedures, type of chemicals used, and establish a monitoring system to assure that the patient care equipment and environment is cleaned, disinfected and stored appropriately so that patient safety is optimized. ${ }^{23-26}$ Staff should be educated on the use of the chemicals and annual competencies are recommended to be required for specific disinfection and sterilization procedures. All cleaning, disinfection and sterilization processes should comply with the CDC guidelines. ${ }^{23}$

High level disinfection and sterilization of patient care equipment is an important area of infection control in many ambulatory settings and is discussed below and is also discussed in detail in Chapter.

\section{Ambulatory surgical centers}

The same aseptic technique and environmental standards apply to all surgical settings. The National Patient Safety Goals (NPSG) for ambulatory and office based surgery provide the elements of performance to achieve safe patient care. ${ }^{27}$ One of the NPSG elements requires improvement in hand hygiene compliance. Another element requires evidence based practices for preventing surgical site infections. The IP can provide guidance to implement the practices. Major components include: hair removal only with clippers and no shaving at the surgical site, administration of antimicrobial agents for prophylaxis for a particular procedure or disease according to guidelines, and education regarding surgical site infection prevention to patients and families as well as staff and licensed independent practitioners involved in surgical procedures. Process and outcome measures need to be documented and reviewed as determined by the ambulatory site's risk assessment. These measures might include incidence of surgical site infection, compliance with antibiotic prophylaxis, time out practices by OR team, or compliance with hair removal methods. The Infection Preventionist can collaborate with the surgery team in identifying the high risk or most frequent surgical procedures performed, so that the surveillance program can be targeted to address key components of operative care. Currently there are no external benchmarks available for SSI or other process measures for surgery performed in ambulatory centers. It is recommended that internal benchmarks be used (ie ambulatory centers track SSI and compliance rates at their institution over time and look for changes over time). Overall surgical infection rates and procedure specific rates (for procedures primarily performed in hospitals), as published by the CDC, may be used.28 
Infection Preventionists and other healthcare workers who clean and process patient care equipment should be familiar with the basic principles for sterilization and disinfection outlined by Earle $\mathrm{H}$ Spaulding which classifies patient care items and equipment into three categories based on the degree of risk of infection involved in the use of the items: critical, semicritical, and noncritical. ${ }^{26}$ This classification provides guidance for determining the level of disinfection required and items requiring sterilization. Flash sterilization, must be kept to a minimum and should not be used as a reason for shortage of instruments per AAMI guidelines. ${ }^{24}$ The Joint Commission Infection Control Standards have now placed a focus on flash sterilization procedures in the OR to assure procedural compliance. ${ }^{25}$ A major challenge to ambulatory surgical centers and the IP is to remain current with changes in sterilization technology and disinfectant products. New surgical equipment is frequently becoming available and manufacturer processing guidelines need to be followed to prevent damage to the instruments as well as adequate disinfection or sterilization.

\section{Safe Injection Practices}

The CDC has implemented recommendations relating to safe injection practices as part of their standard precautions to provide safety to both the healthcare worker and patient. Injuries due to needles and other sharps have been associated with the transmission of hepatitis $\mathrm{B}$ virus, hepatitis $\mathrm{C}$ virus and human immune deficiency virus. The federal Needlestick Safety and Prevention Act signed into law in November 2000 authorized OSHA's revision of its Bloodborne Pathogens Standard to require the use of safetyengineered sharp devices. ${ }^{29}$ Ambulatory services need to provide policies and infection control in-services to staff regarding aseptic technique and correct management of sharps in patient care.

Syringes, needles, insulin pens and all sharps must be single use. Changing a needle or cannula and using the same syringe may still support transmission of pathogens or viruses. Fluid infusion and administration should be single use. Single dose vials should be used whenever possible and clearly dedicated to a single patient. If multiple dose vials are used they must be stored appropriately according to manufacturer recommendations and kept in the immediate patient treatment areas. Bags or bottles of intravenous solution used as a common source for multiple patients is an unsafe practice and has been associated with outbreaks. ${ }^{30}$

Inappropriate use of product among multiple patients has resulted in many large and harmful outbreaks. Examples include a hepatitis B outbreak in New York City in 2001 correlated to multidose use for injections and contaminated syringes left on the table where medications were prepared hepatitis C outbreak in a Nevada Endoscopy clinic in 2007 due to re use of same syringe for propofol administration; and re-use of glucometers and insulin pens resulting in hepatitis $\mathrm{B}$ and $\mathrm{C}$ transmission. ${ }^{31-33}$

\section{Bioterrorism and Disaster Planning}

A risk assessment of the clinic location and the community it services provides guidance for emergency preparedness emergency plans. Factors to consider in planning are the socioeconomic level and vaccine status of the population, natural disasters the area may be prone to, and access routes to the clinic. Clinics associated with health systems should be included in the emergency plans of the health system. Independent clinics need to collaborate with a local hospital, public health department and government agencies. The plans should provide a process by which the facility can use mitigation strategies to become prepared to respond to and recover from all types of disasters. Infection Preventionists should actively participate in the planning process. ${ }^{34}$ 


\section{Conclusion}

Outpatient services are continually increasing and changing with expansion of new technologies. Increased use of invasive devices and procedures provides new and challenging risks for infection. Risks associated with contaminated equipment can be decreased by knowledge and maintenance of aseptic technique and disinfection practices. The challenge to infection control and ambulatory staff is to remain updated and familiar with these emerging technologies to increase the likelihood of preventing healthcare associated infection and providing safe patient care. More patients with a high acuity of illness are now being seen in ambulatory instead of inpatient settings, and these patients often spend prolonged periods in waiting rooms, in close proximity to others. The risk of communicable disease transmission and presence of multiple drug resistant organisms necessitates standard and transmission-based precautions for all patient care settings, including ambulatory settings. The healthcare worker, patient and family members of patients need to be provided education to support patient safety and minimize risk for infection. The Infection Preventionist has unique challenges in providing the ambulatory clinic both infection prevention and regulatory strategies.

\section{REFERENCES}

1. Smith PW, Bennett G, Bradley SF, et al. Infection prevention and control in long-term care facilities. Infect Control Hosp Epidemiol. 2008; 29:785-814. [PubMed: 18767983]

2. Friedman C, Barnette M, Buck AS, et al. Requirement for infrastructure and essential activities of infection control and epidemiology in out-of-hospital settings: A consensus panel report. Infect Control Hosp Epidemiol. 1999; 20:695-705. [PubMed: 10530650]

3. Jarvis WR. Infection control and changing health-care delivery systems. Emerg Infect Dis. 2001; 7:170-173. [PubMed: 11294699]

4. Coleman EA. Falling through cracks: Challenges and opportunities for improving transitional care for persons with continuous complex care needs. J Am Geriatr Soc. 2003; 51:549-555. [PubMed: 12657078]

5. Aiello A, Malinis M, Knapp J, Mody L. Hand hygiene practices in nursing homes: Does knowledge influence practice? Am J Infect Control. 2009; 37:164-167. [PubMed: 18945512]

6. Mody L, McNeil SA, Sun R, et al. Introduction of a waterless alcohol-based hand rub in a long-term care facility. Infect Control Hosp Epidemiol. 2003; 24:165-171. [PubMed: 12683506]

7. Pittet D, Sax H, Hugonnet S, et al. Cost implications of successful hand hygiene promotion. Infect Control Hosp Epidemiol. 2004; 25:264-266. [PubMed: 15061421]

8. Centers for Disease Control and Prevention. Guideline for hand hygiene in healthcare settings: Recommendations of the Healthcare Infection Control Practices Advisory Committee and the HICPAC/SHEA/APIC/IDSA Hand Hygiene Task Force. MMWR. 2002; 51:S3-S40.

9. Pittet D, Allegranzi B, Boyce J. The World Health Organization guidelines on hand hygiene in health care and their consensus recommendations. Infect Control Hosp Epidemiol. 2009; 30:611622. [PubMed: 19508124]

10. Mody L, Saint S, Kaufman S, Kowalski C, Krein S. Adoption of alcohol-based handrub by United States hospitals: a national survey. Infect Control Hosp Epidemiol. 2008 Dec.29:1177-1180. [PubMed: 18986300]

11. Pedersen LK, Held E, Johansen JD, et al. Less skin irritation from alcohol-based disinfectant than from detergent used for hand disinfection. Br J Dermatol. 2005; 153:1142-1146. [PubMed: 16307649]

12. Bradley SF. Issues in management of resistant bacteria in long-term care facilities. Infect Control Hosp Epidemiol. 1999; 20:362-366. [PubMed: 10349960]

13. Hujer AM, Bether CR, Hujer KM, et al. Antibiotic resistance in the institutionalized elderly. Clin Lab Med. 2004; 24:343-361. [PubMed: 15177844]

14. Goldrick BA. MRSA, VRE and VRSA: How do we control them in nursing homes? Emerg Infect. 2004; 104:50-51.

Infect Dis Clin North Am. Author manuscript; available in PMC 2012 March 1. 
15. Muto CA, Jernigan JA, Ostrowsky BE, et al. SHEA guideline for preventing nosocomial transmission of multidrug-resistant strains of Staphylococcus aureus and enterococcus. Infect Control Hosp Epidemiol. 2003; 24:362-386. [PubMed: 12785411]

16. Siegel, JD.; Rhinehart, E.; Jackson, M., et al. Management of multi-drug resistant organisms in healthcare settings. 2006. http://www.cdc.gov/ncidod/dhqp/pdf/ar/MDROGuideline2006.pdf

17. Siegel, JD.; Rhinehart, E.; Jackson, M., et al. Guideline for isolation precautions: preventing transmission of infectious agents in healthcare settings. 2007. http://www.cdc.gov/ncidod.dhqp/pdf/isolation2007.pdf

18. Horan, TC.; Gaynes, RP. Surveillance of nosocomial infections. In: Glen Mayhall, C., editor. Hospital Epidemiology and Infection Control. 3rd Ed. Philadelphia, PA: Lippincott Williams \& Wilkins; 2004. p. 1661-1702.

19. McGeer A, Campbell B, Emori TG, et al. Definitions of infection for surveillance in long-term care facilities. Am J Infect Control. 1991; 19:1-7. [PubMed: 1902352]

20. Stevenson KB, Moore J, Colwell H, et al. Standardized infection surveillance in long-term care: Interfacility comparisons from a regional cohort of facilities. Infect Control Hosp Epidemiol. 2005; 26:231-238. [PubMed: 15796273]

21. Embril JM, McLeod JA, AL-Barrak AM, et al. An outbreak of methicillin-resistant Staphylococcus aureus on a burn unit: potential role of contaminated hydrotherapy equipment. Burns. 2001; 27:681-688. [PubMed: 11600247]

22. Centers for Disease Control and Prevention. Atlanta: Centers for Disease Control and Prevention; 2005. Guideline for Preventing the Transmission of Mycobacterium Tuberculosis in Healthcare Settings.

23. Rutala, D., W., editor. Centers for Disease Control and Prevention. Guideline for Disinfection and Sterilization in Healthcare Facilities. Weber Infection Control Practices Advisory Committee (HICPAC); 2008.

24. Comprehensive Guide to Steam Sterilization and Sterility Assurance in Health Care Facilities. Association for the Advancement of Medical Instrumentation. 2006 July.

25. Steam Sterilization -Update on The Joint Commission's Position. www.jointcommission.org/Library/WhatsNew/steam_sterilization.htm

26. Spaulding, EH. Chemical disinfection of medical and surgical materials. In: Lawrence C Block, SS., editor. Disinfection sterilization and preservation. Philadelphia: Lea \& Febiger; 1968. p. 517-531.

27. Ambulatory Health Care Accreditation Program 2010 Chapter: National Patient Safety Goals. The Joint Commission on accreditation of Healthcare Organizations. www.jointcommission.org/standards

28. National Healthcare Safety Network (NHSN) report: Data summary fodr 2006 through 2008 , issued December 2009. Vol. 37. Association for Professionals in Infection Control and Epidemiology, Inc.; 2009. p. 783-805.

29. Needlestick Safety and Prevention Act (PL 106-430). 2000 Nov 6.

30. Morbidity and Mortality Weekly Report. Vol. Vol.52. 2003 September 26. Transmission of Hepatitis B and C Viruses in Outpatient Settings-New York, Oklahoma, and Nebraska, 20002002.

31. Infection Control and Hospital Epidemiology. Vol. 26. 2005. Hepatitis B outbreak in New York City, 2001; p. 745-760.

32. Morbidity and Mortality Weekly Report. Vol. Vol.57. 2008 May 16. Acute Hepatitis C Virus Infections Attributed to Unsafe Injection Practices at an Endoscopy Clinic-Nevada, 2007.

33. U.S. Food and Drug Administration Alert. FDA: Insulin Pens and Insulin Cartridges Must Not Be Shared. 2009 March 19.

34. Emergency Management Chapter 117 and Chapter 118 Infectious Disease Disasters: Bioterrorism, Emerging Infections, and Pandemics. APIC Text of Infection Control and Epidemiology $3^{\text {rd }}$ Edition Volume II Scientific and Practice Elements 2009. 


\section{Table 1}

\section{Resources for Infection Preventionists}

1. Society for Healthcare Epidemiology of America (SHEA) and the Association for Professionals in Infection Control (APIC) both have longterm care committees that publish and approve SNF infection guidelines and publish periodic position papers related to pertinent infection control issues. Their websites have several educational resources for staff education and in-services. In addition, APIC also publishes a quarterly long-term care newsletter.

2. Local APIC chapters provide a network for infection control practitioners to socialize, discuss infection control challenges and practical solutions to overcome them, and provide access to educational resources and services. Infection control practitioners should become members of APIC at both the local and national level to remain up-to-date with practice guidelines, position statements, information technology resources, and changes in policies and regulations.

3. Hospital Epidemiology and Infection Control, $3^{\text {rd }}$ Ed.; C. Glen Mayhall Ed.: Lippincott Williams \& Wilkins: Philadelphia, PA.

4. Selected Internet Websites:

a. Centers for Disease Control and Prevention (CDC): http://www.cdc.gov

b. Society for Healthcare Epidemiology of America (SHEA): http:// www.shea-online.org/

c. Association for Professionals in Infection Control (APIC): http://www.apic.org

d. Occupational Health and Safety Administration (OSHA): http://www.osha.gov

e. Joint Commission on Accreditation of Healthcare Organizations-Infection Control Initiatives:

http://www.jcaho.org/accredited+organizations/patient+safety/infection+control/ic+index.htm 